\title{
ÜBER VORKOMMEN UND BEDEUTUNG VON TERPSINOE AMERICANA (BAIL.) RALFS IN DEN ABLAGERUNGEN DES LITORINAMEERES
}

\author{
PENTTI ALHONEN, AARRE HEINO und RISTO TYNNI
}

\begin{abstract}
ALHONEN, P.; HEINO, A. und TYNNI, R., 1984. Über Vorkommen und Bedeutung von Terpsinoe americana (Bail.) Ralfs in den Ablagerungen des Litorinameeres. Bull. Geol. Soc. Finland 56, Part 1-2, 117-133.

In diesem Artikel werden das Vorkommen und die Bedeutung einer subfossilen Kieselalgenform Terpsinoe americana (Bail.) Ralfs in den Sedimenten des Litorinameeres diskutiert. In den letzten Jahren sind in Finnland neue Beobachtungen über die Art gemacht worden. Obwohl Terpsinoe americana in der Entwicklungsgeschichte der Ostsee selten ist, kommt sie in manchen Probeserien sowohl in den Benthos-Epiphytenassoziationen als zusammen mit den planktischen Diatomeen vor. Als typische ozeanische Art kann Terpsinoe americana als Vertreter des Höhepunkts der Salinität und der Abkühlung des Klimas nach dem Ende der postglazialen Wärmezeit gelten. Die systematische Stellung der Art und ihre heutige Ökologie werden auch diskutiert. Die Schalenstruktur von Terpsinoe americana des finnischen Materials wurde mit der SEM-Technik untersucht, und sie entspricht deutlich den Grundstrukturtypen der Biddulphiaceae-Diatomeen.
\end{abstract}

\begin{abstract}
The subject of this paper is the occurrence and significance of the subfossil diatom Terpsinoe americana (Bail.) Ralfs. New observations of this species have recently been made in Finland. Although Terpsinoe americana is rare in the developmental history of the Baltic Sea, it occurs in many cores in the benthonicepiphytic community and among the planktonic diatoms. As a typical oceanic species, Terpsinoe americana is related to the maximum salinity level and temperature of the Litorina Sea, although it probably lived in the ecological periphery of the sea. The disappearance of this species coincides with the decrease in salinity of the Baltic Sea and the end of the Holocene Climatic Optimum. Taxonomical problems and the recent ecology of Terpsinoe americana are also discussed. The valvar structure of the species in the Finnish material presented in this paper was examined by SEM and found to be consistent with that of the diatoms of the family Biddulphiaceae.
\end{abstract}

Key words: Litorina Sea, Terpsinoe americana, diatoms.

Pentti Alhonen: Department of Geology, University of Helsinki, P.O. Box 115, SF-00171 Helsinki 17, Finland.

Aarre Heino: Department of Geography, University of Turku, 20500 Turku 50.

Risto Tynni: Geological Survey of Finland, Kivimiehentie 1, SF-02150 Espoo 15.

\section{Einleitung}

Als eine ausgeprägte, leicht erkennbare Diatomeenart ist Terpsinoe americana (Bail.) Ralfs ein passendes Forschungsobjekt ihrer Verbreitung, Ökologie und Entwicklungsgeschichte. Im Bereich der nördlichen Ostsee ist ihr Vorkommen als thermophile Indikatorart im Quartär 
zweifellos aussergewöhnlich. In den Landhebungsgebieten der Küste wurde diese Art schon früher in einigen Ablagerungen des Litorinameeres gefunden (Aario 1932, Нyyppä 1937, Cleve-Euler 1951, Heino 1973, Alhonen 1974 und Eronen et al. 1982). Terpsinoe americana wurde auch in einem Lehmklumpen aus der Eemperiode in Rouhiala angetroffen (Brander 1937). Ihr Vorkommen in einem kleinen Teich in Sondby bei Porvoo (Fontell 1926) kann mit der Erosion von Sedimenten des Litorinameeres verbunden sein, denn bei den Kontrolluntersuchungen konnte man die Art dort nicht mehr finden. Andererseits sind Beobachtungen aus den südlichen und nördlichen Teilen der Ostsee sehr selten, und Kenntnisse über rezentes Vorkommen können nicht immer als unbestreitbare Beweise der heutigen Ausbreitung dieser Diatomeen dienen. Die Funde leerer Schalen aus den Oberflächensedimenten der Ostsee können nämlich von der Erosion der Ablagerungen herrühren.

Terpsinoe americana ist eine kosmopolitische Kieselalge mit vielseitigen ökologischen Angaben. Bei der Betrachtung ihrer Anpassungsfähigkeit an verschiedenartige Umweltverhältnisse wurde vor allem Rücksicht auf die Temperatur und den Salzgehalt des Wassers genommen. Wenn die zur Erhaltung der Diatomeenarten beitragenden Faktoren im allgemeinen geschätzt werden, ist ihre heutige Ökologie ein wichtiger Ausgangspunkt. Als Masstab der Adaptation dient dann das Begriffspaar Eurytopie/Stenotopie angewandt mit den Variationen von Temperatur und Salzgehalt (Simonsen 1962). Für solche Betrachtungen sind die Diatomeen mit ihren gut haltbaren Formen und grosser Artenstatistik sehr gut geeignet. Die Deutung wird jedoch durch eine mögliche mit der Evolution zusammenhängende Veränderung im ökologischen Verhalten erschwert. Ihre Bedeutung ist wohl recht gering bei den marinen Diatomeen. Denn das Meer vertritt bekanntlich erhaltende Verhältnisse und in dieser Umgebung ist der Veränderungsdrang der ökologischen Anforderun- gen vermutlich sehr gering.

Die frühe Meeresumgebung von Terpsinoe americana im Oligozän und Miozän hat sich vermutlich nicht viel verändert. Am ähnlichsten sind heute die Verhältnisse im Indischen Ozean und in den Fernostmeeren der Sowjetunion, wo immer noch zahlreiche Reliktendiatomeen aus dem Miozän und Pliozän vorkommen. Auch in anderen Meeren können im Tertiär entstandene Diatomeenformen heute noch gefunden werden, obwohl in geringer Zahl.

\section{Artenidentifizierung und Systematik}

Nach Hustedt (1930) sind die meisten Kennzeichen von Terpsinoe americana konstant. Zahlreiche lichtmikroskopische Untersuchungen liegen vor. Von ihnen sollen besonders die folgenden erwähnt werden: Schmidt (1895, pl. 200, figs. 9-13), Peragallo (1908, pl. 90, figs. 5 und 6), Boyer (1916, pl. 6, fig. 10; 1926, pt 1, p. 145), Brockmann (1928, pl. 3, fig. 8), Hustedt (1930, pt 1, p. 900, fig. 541), ProschkinaLavrenko (1949, pt 1, p. 169, pl. 10, fig. 3), Cleve-Euler (1951, pt 1, p. 128, fig. 288) und Riznyk (1973, pl. 16, figs. 8 und 9). Die Beispiele unseres Materials sind auf den Abbildungen 1-3 dargestellt. In der länglichen Schale von Terpsinoe americana können die apikalen Enddörnchen und an beiden Seiten drei transapikale Dörnchen unterschieden werden. An beiden Seiten des mittleren Dörnchens gehen von der Oberfläche ziemlich tief eindringende Septen aus. Die Enddörnchen unterscheiden sich von der übrigen Oberflächenstruktur als strukturlos. Die Oberflächenstruktur besteht in einer strahlenförmigen Punktierung. Das Mittelfeld hat keinen mit einer kegelförmigen Pore versehenen Höcker, was sie von $T$. musica unterscheidet. An den Seitenwänden kann man eine ähnliche Musterung wie an der Valvarebene sehen. Die Richtung ist dieselbe wie bei der Pervalvarachse.

In der Literatur gibt es weniger elektronenmikroskopische als optische Beschreibungen 


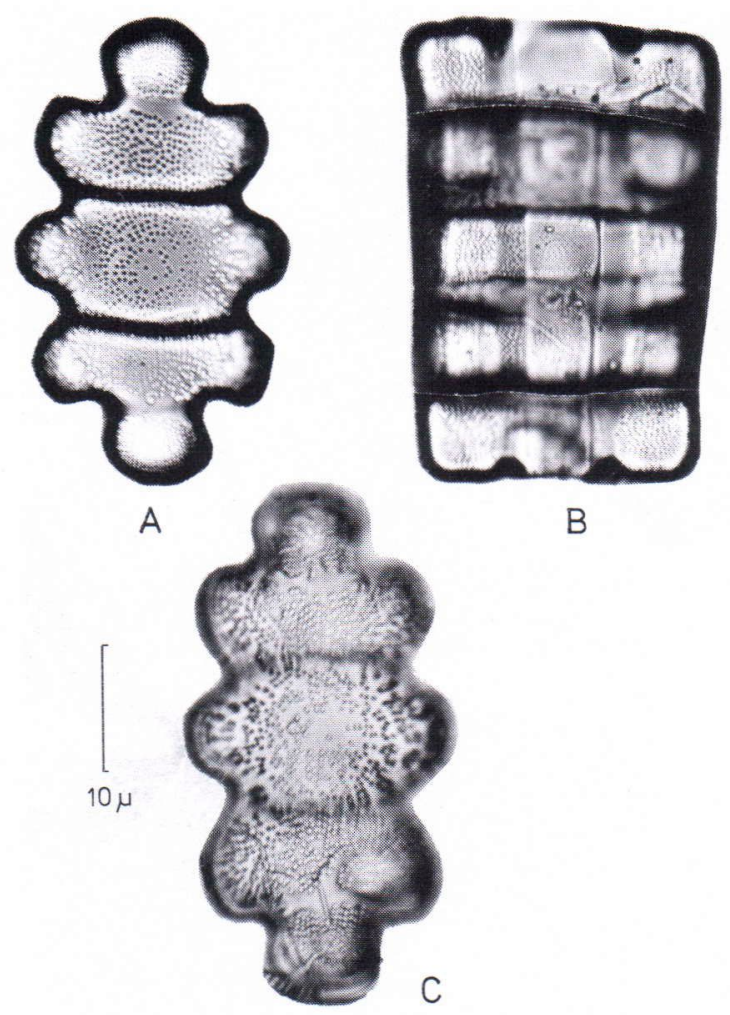

Abb. 1. Die Schale von Terpsinoe americana. A. Probeserie 5/72, B. Gürtelbandansicht (Probeserie 7/72), C. Tuorsniemi, Pori (Alhonen 1974).

Fig. 1. Frustule of Terpsinoe americana. A. Core 5/72, B. Girdle view (core 7/72), C. Tuorsniemi, Pori (Alhonen 1974).

über Terpsinoe americana. Von den ersteren soll die Darstellung von Okuno (1959, pl. 21, fig. 1) über die 12500 mal vergrösserte Valvarebene von $T$. americana erwähnt werden. Nach Okuno (op. cit.) liegen die Poren der Schale »locular, closed outwards by a thin irregularly porous sieve membrane, and opens (almost freely?) inwards with a round opening about $200-$ $400 \mu$ in diameter. In many valves the sieve membranes are well preserved».

Ross und Sims (1971 und 1972) haben ausführlichere SEM-Untersuchungen über die zur Familie Biddulphiaceae gehörenden Gattungen durchgeführt und eine Terminologie für die Identifizierung der entblössten Strukturmuste- rungen entwickelt. Die Grundstrukturtypen der Schalen der Biddulphiaceae-Diatomeen sind porös und wabenartig. Die Struktur von Terpsinoe entspricht dem erstgenannten Typ, was auch deutlich auf den SEM-Bildern des finnischen Materials hervortritt (Abb. 2-3).

Die Erläuterung der Systematik von Terpsinoe americana ist verhältnismässig kompliziert. $\mathrm{Zu}$ den früheren Untersuchungen gehören diejenigen von Hustedt (1930) sowie Ross und Sims (1971). In Hustedt's Klassifizierung (1930) hat man Schütts (1896) Systematik gefolgt; da gehört T. americana zur Familie Anauleae. Dazu werden auch die nahen Gattungen Anaulus Ehr. und Eunotogramma Weisse klassifiziert. Die Familie Anauleae gehört in dieser Klassifizierung zur Gruppe Biddulphioideae Schütt hauptsächlich aufgrund der Struktur der Schale. Die Anwendung des Elektronimikroskops hat danach vor allem die Kenntnis der Schalenstruktur der Diatomeen gefördert und zu taxonomischen Präzisierungen geführt, besonders seitdem die bei Fossielienuntersuchungen festgestellte phylogenetische Entwicklung im höheren Grade berücksichtight worden ist.

Ross und Sims (1971) habe festgestellt, dass die Gattung Terpsinoe Ehr. strukturell zur Familie Biddulphiaceae gehört, die in zwei Unterfamilien: Hemiauloideae und Biddulphioideae verteilt ist. Zur letztgenannten gehört auch die Gattung Terpsinoe. Simonsen (1972 und 1979) befolgt in seiner Systematik dieselbe Verteilung, und diese Auffassung ist wohl heute allgemein anerkannt. Im folgenden werden Beobachtungen über diese Verteilung mit Rücksicht auf die Gattungen Terpsinoe, Hydrosera, und Triceratium behandelt.

Nach Simonsen (1979) hat die Hydrosera einen »labiate process subcentral». Bei $H$. triquetra ist er deutlich erkennbar, aber die in Finnland vorkommende $H$. trifoliata besitzt dieses taxonomische Kennzeichen nicht (Tynni 1977). Cleve (1881) hat eine Familienähnlichkeit von allem zwischen Hydrosera trifoliata und Triceratium gefunden. Brun (1891) hat diese 

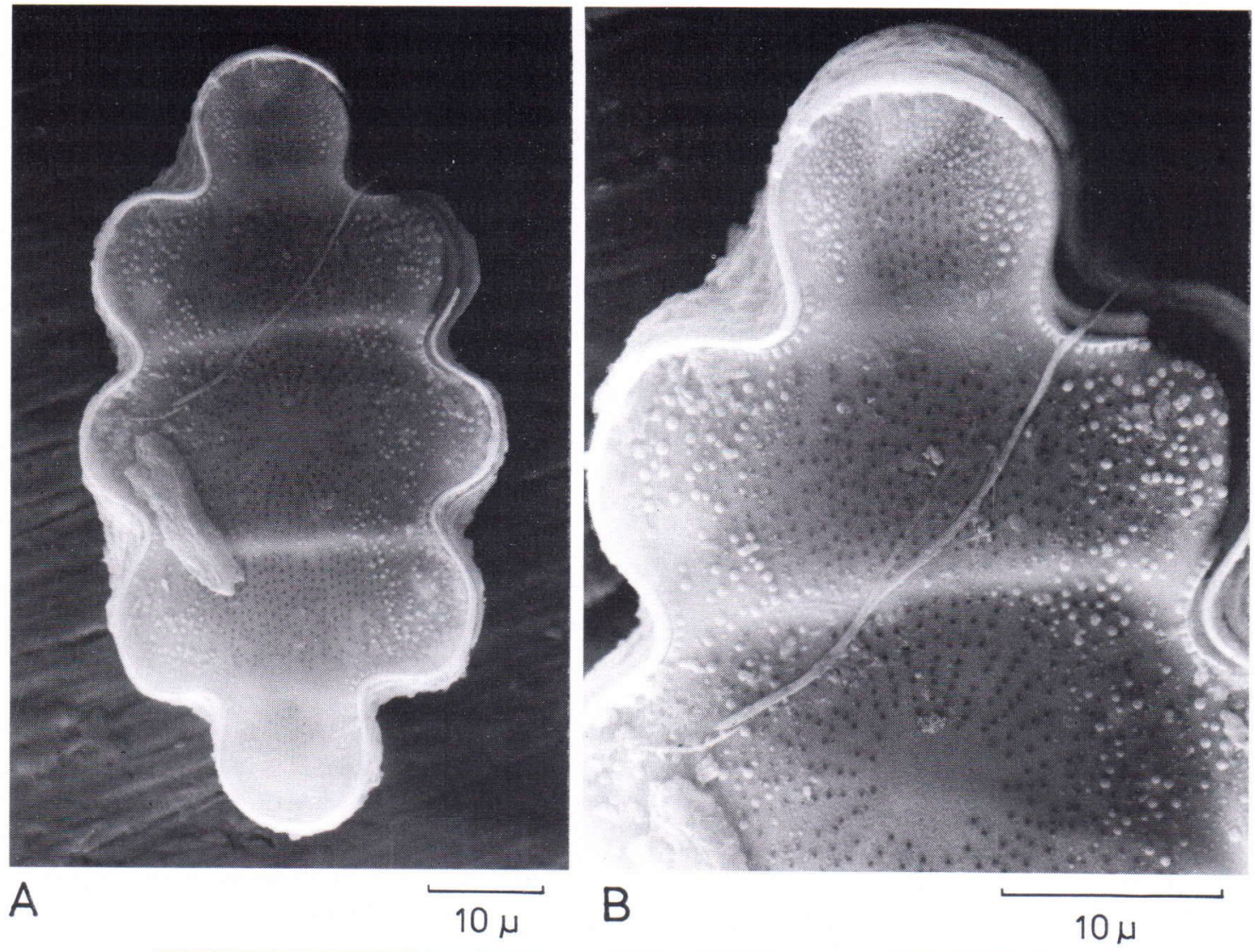

A
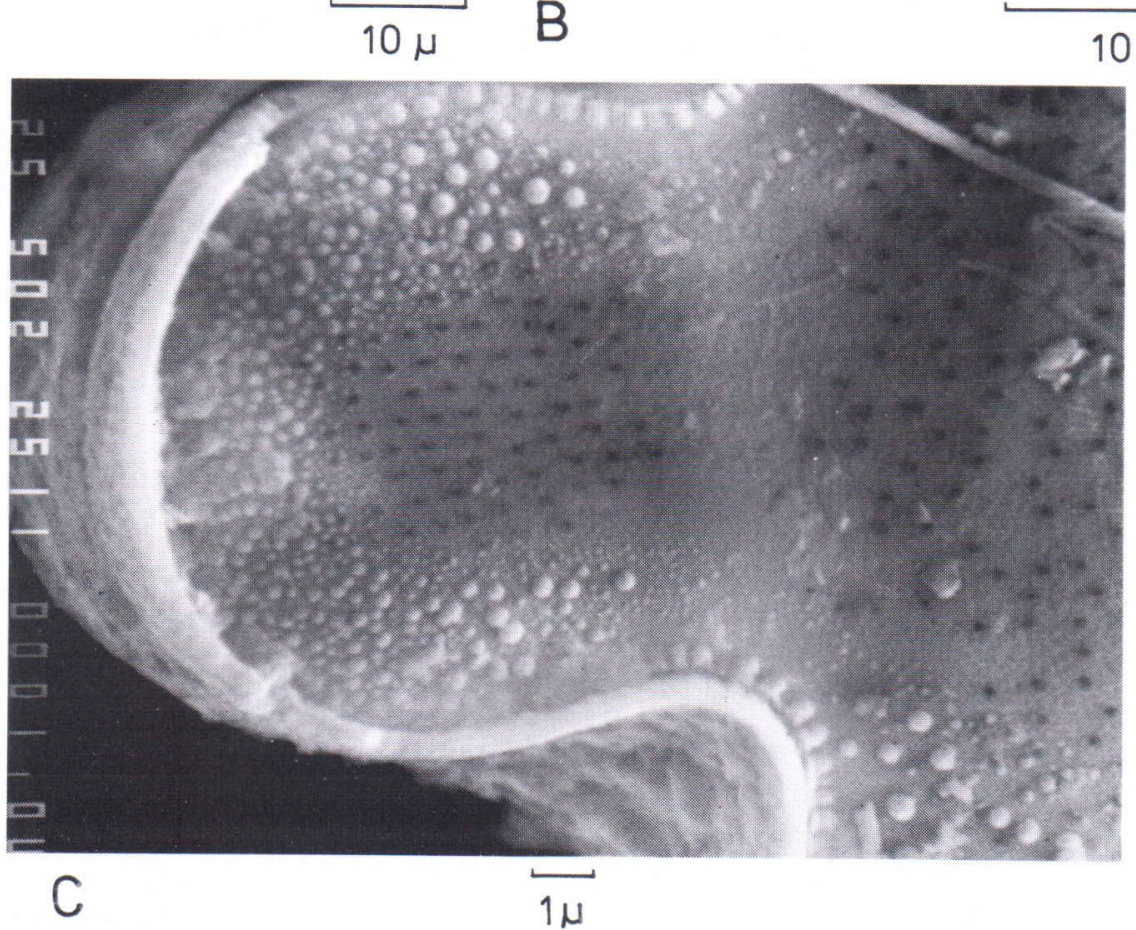
Art mit der Gattung Terpsinoe ( $T$. inflata Brun) verbunden. Auch Cleve-Euler (1915) hat sich dieser Auffassung angeschlossen, aber später (1951) hat sie diese Art zur Gattung Triceratium geführt. Nach unserer Meinung gehört Hydrosera trifoliata nicht zu Terpsinoe, denn die strukturellen Unterschiede sind eigentlich verhältnismässig gross. Erstens sind die anderen Arten der Gattung Terpsinoe bipolar, und zweitens ist die Struktur der Ecken der TerpsinoeArten feiner als die der übrigen Schale und drittens dringen die Septen oder Costae der Terpsinoe tief in die inneren Teile. Hydrosera trifoliata hat dagegen nur rudimentäre keilförmige Dörnchen (Tynni 1977, Taf. 4, E). Nach Heurck ist die Form der Schale von Hydrosera Wallich dreieckig mit Falten an den Enden. Die Oberflächenstruktur ( $H$. triquetra) ist zellengewebeartig und die Seitenfigur ist viereckig. Die Membrane ist and den Seiten feinpunktiert. Die Schalen bilden Bänder. Nach Hustedt (1930, p. 900) ist $H$. triquetra eine selbständige zur Gattung Hydrosera gehörende Art, die nicht zur Gattung Terpsinoe klassifiziert werden kann. Ökologisch ist sie sehr anpassungsfähig (Simonsen 1965) und kommt auch im Süsswasser vor. Hydrosera triquetra hat jedoch gewisse Ähnlichkeiten mit $H$. trifoliata. Die Schale ist dreieckig und hat Endfalten. Die Ecken unterscheiden sich vom Mittelfeld als keilförmige Höcker. Im fossilen Material kommen auch bandförmige Kolonien vor. Die Art ist offenbar ökologisch anpassungsfähig gewesen.

In diesem Zusammenhang wird Triceratium Ehr. als die bei Hustedt (1930) beschriebene Art angesehen. In Manns (1907) Klassifizierung entspricht die Gattung Trigonium den Diatomeenformen, bei denen die Buckel der Schalenecken rudimentär sind. Andrerseits sind viele ursprünglich zu Triceratium klassifizierte Arten nach Simonsen (1979) zur Gattung Stictodiscus versetzt worden. Die Form der Schale ist dreieckig oder polygon und nur ausnahmsweise bipolar oder rund, wobei das Polygon an den Buckeln vorkommt. Sie können auch reduziert sein, und ihre Struktur ist im allgemeinen von der übrigen Schalenstruktur abweichend, und sie formen oft zylindrische, diagonale Eckenanhänge. Der wichtigste Unterschied ist das Fehlen der Eckenbuckel bei Hydrosera. Ihre Ekkenstruktur ist auch nicht von derjenigen der Valvarebene abweichend. Bei der Gattung Terpsinoe ist dagegen die Struktur der epikalen Ekken feiner an der übrigen Valvarebene. In dieser Hinsicht ist Terpsinoe taxonomisch näher der Triceratium als der Hydrosera.

\section{Ökologische Beobachtungen}

Das heutige Vorkommen von Terpsinoe americana ist weltweit, obwohl nicht sehr ausgiebige Beobachtungen vorliegen. In vielen Fällen geht es um Einzelfunde, die durch Wellenschlag oder Strömungen von ihrem Standort getrennt sind. Auf Abbildung 4 sind mehrere rezente Fundstellen von Terpsinoe americana dargestellt um die Ausbreitung dieser Art klarzulegen. Die Beobachtungen konzentrieren sich auf geschützte Küstengebiete der subtropischen und tropischen Zonen. Im Zusammenhang mit Planktonuntersuchungen begegnet man ihnen meistens nicht. Die meisten Fundstellen von Terpsinoe americana liegen zwischen $45^{\circ}$ nördlicher und südlicher Breite.

In der allgemeinen ökologischen Interpretation ist Terpsinoe americana eine in der tropischen und gemässigten Zone wachsende Epiphytform, die nur im Salz- und Brackwasser gedeiht. Als

Abb. 2. Terpsinoe americana. Elektronenmikroskop-Bilder von der Schalenstruktur mit verschiedenen Vergrösserungen $(\mathrm{A}-\mathrm{C})$.

Fig. 2. Terpsinoe americana. SEM of frustule with different magnifications $(\mathrm{A}-\mathrm{C})$. 

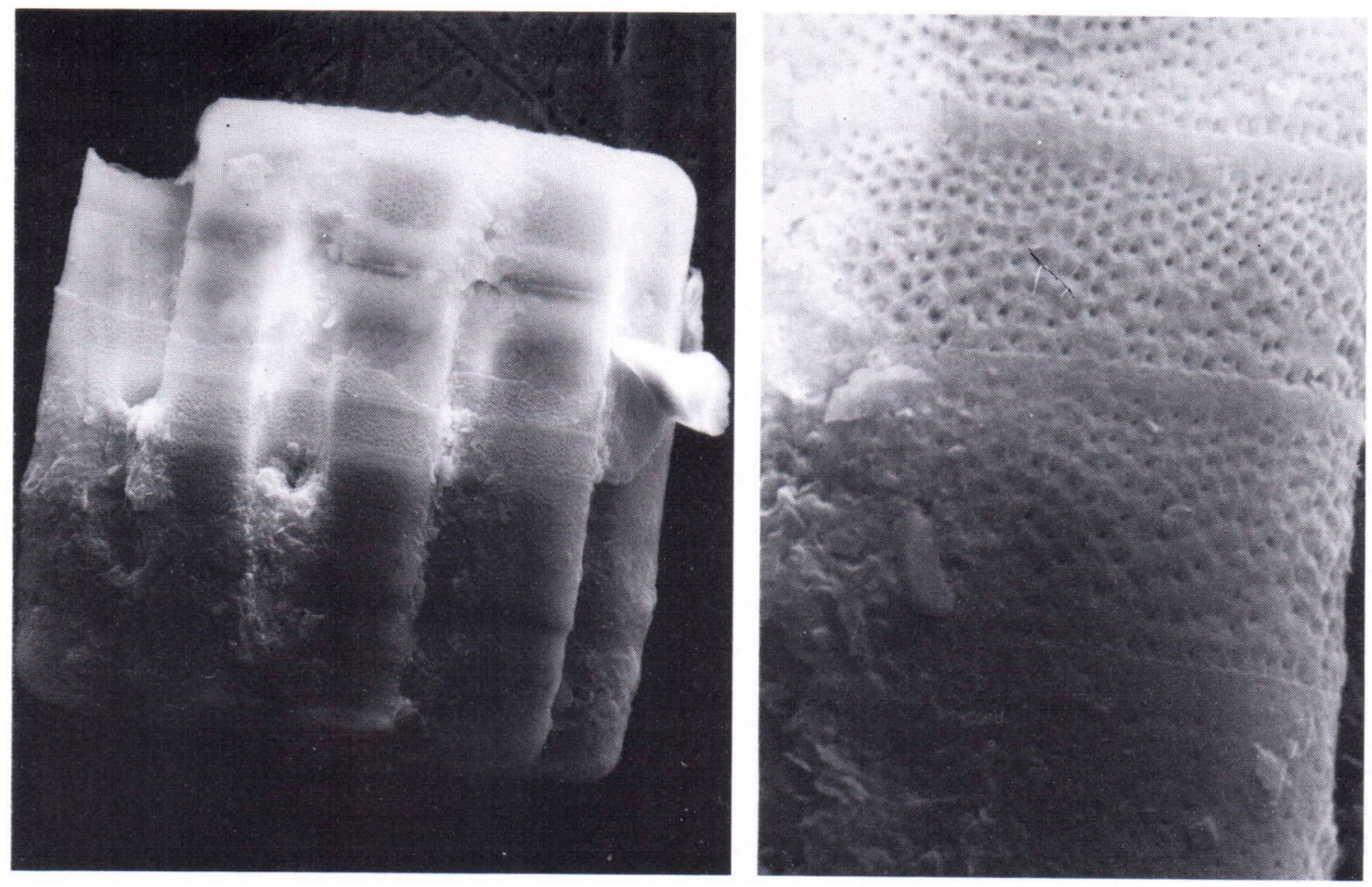

A
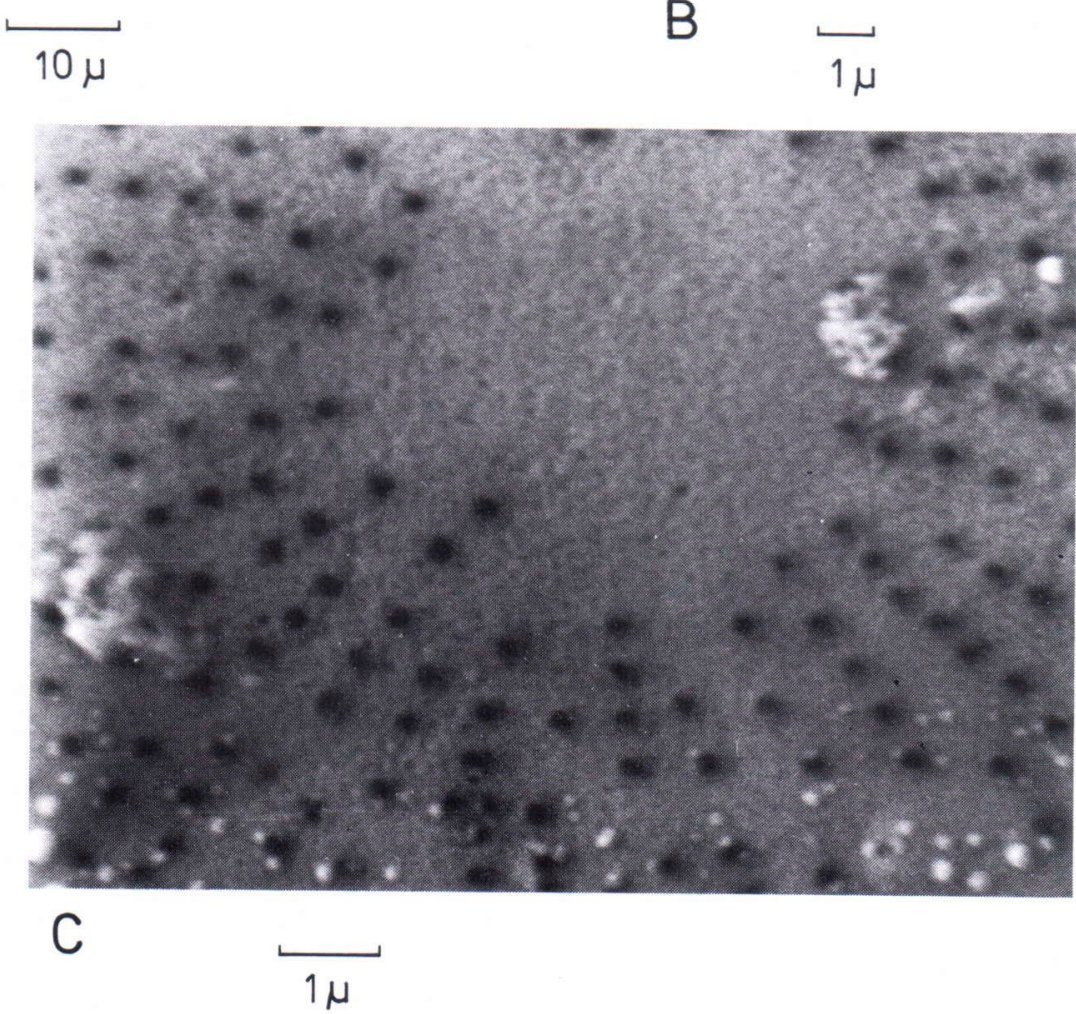


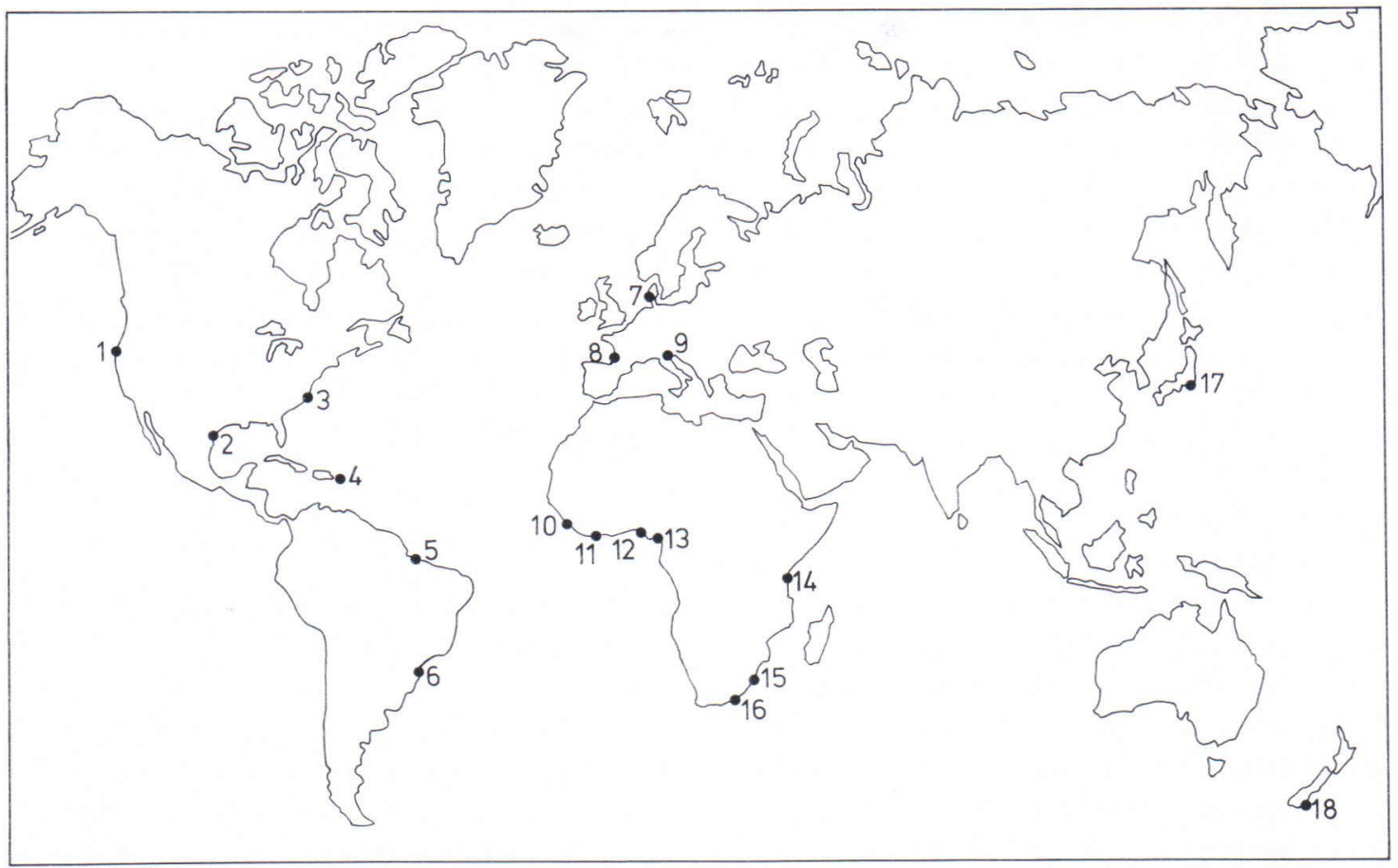

Abb. 4. Das rezente Vorkommen von Terpsinoe americana. 1. siehe Riznyk (1973), 2. Wood (1963), 3. Hustedt (1955), 4. Hagelstein (1938), 5. Hustedt (1930), 6. Moreira (1960), 7. Brockmann (1950), 8. H. et M. Peragallo (1897-1908), 10-12. Woodhead und Tweed (1960), 13. Woodhead und Tweed (1958), 14. Amosse (1924), 15. Cholnoky (1968), 16. Giffen (1970), 17. Schmidt (1874-) und 18. Taylor (1970).

Fig. 4. Recent occurrence of Terpsinoe americana. For further details see references.

gutes Beispiel für ihre heutige Ökologie dient Yaquina Estuary in Oregon (ca. $44^{\circ}$ nördl. Br.), die Riznyk (1973) untersucht hat. Im Delta des Flusses Yaquina ist der Einfluss von Ebbe und Flut ca. $1.67 \mathrm{~m}$.

Die Temperatur der Oberfläche des Sediments variiert von $32.5^{\circ} \mathrm{C}$ im Juli bis $5^{\circ} \mathrm{C}$ im Februar während der Ebbe. Die Grenzwerte sind Temperaturen der entblössten Sedimentoberflächen. Auf wasserbedeckten Gebieten sind die entsprechenden Variationen $15^{\circ} \mathrm{C}-9^{\circ} \mathrm{C}$.
Die Variationen im Salzgehalt des Deltas sind auch gross: im Sommer 33-35 o/oo und im Winter $8 \mathrm{o} / \mathrm{oo}$. In der beschriebenen Umgebung ist Terpsinoe americana allgemein auf der Oberfläche des Schliffsediments von Sally's Bend, und sie wurde auf der mittleren und oberen Flut - und Ebbezone das ganze Jahr lang beobachtet. Dies deutet auf eine eurytopische Salz- und Temperaturökologie dieser Art hin. Ein zweites Beispiel für die Ökologie von Terpsinoe americana ist die Untersuchung von

Abb. 3. Terpsinoe americana. Elektronenmikroskop-Bilder von der Schalenstruktur. A. Gürtelbandansicht, B. Detallierte Struktur des Gürtelbandes, C. Struktur des Mittelfelds.

Fig. 3. Terpsinoe americana. SEM of frustule. A. Girdle view, B. Details of the connecting band, C. Structure of the central area. 
König (1959) über die Diatomeenflora der Arcachon Bucht. Hier bildet diese Art einen bedeutenden Teil der Gesamtartenzusammensetzung, Peragallo (1897-1908) hat sie als erster gefunden. Die geschützte Arcachon Bucht liegt südlich vom $45^{\circ}$ Breitegrad südwestlich von Bordeaux. Das Areal der Bucht ist ca. $160 \mathrm{~km}^{2}$ und der Einfluss von Ebbe und Flut ist dort $3.5 \mathrm{~m}$. Das Oberflächensediment des Bodens ist hauptsächlich Sand, aber an sedimentologisch ruhigeren Stellen kommt Ton und organisches Material vor. In der Bodenmorphologie sind die von der Flut bedeckten Watt-Gebiete und die Strömungstiefen zwischen ihnen vertreten. Der Salzgehalt des Wassers variiert an der südlichen Küste der Arcachon Bucht zwischen 33.8 und 19.9 o/oo. Näher an der Mündung des LeyerFlusses ist der winterliche Salzgehalt bedeutend niedriger, aber auch dort kommt Terpsinoe americana vor. Die durchschnittliche Temperatur in der Arcachon Bucht variiert zwischen $7^{\circ} \mathrm{C}$ (Januar) und $22^{\circ} \mathrm{C}$ (Juli).

König (1959) vergleicht die Temperaturen im Wattenmeergebiet in Nord-Deutschland mit den Werten in der Arcachon Bucht sowie die Diatomeenzusammensetzung der beiden Gebiete. Der durchschnittliche Salzgehalt im norddeutschen Wattenmeergebiet, $30 \mathrm{o} / \mathrm{oo}$, ist fast derselbe wie in der Arcachon Bucht. Die Temperatur ist dagegen im Meergebiet der Insel Sylt monatlich $4-10^{\circ} \mathrm{C}$ niedriger als in Arcachon. Eine rezente Terpsinoe americana-Kultur konnte nur in Arcachon gefunden werden. Während der mit dem Litorinameer ähnlichen Nordsee-Diluvium-Periode war diese Art allgemein an der Nordsee-Küste und Brockman (1928 und 1940) hat sie deswegen als ein Leitfossil dieser Periode vorgeschlagen.

Ihr Temperaturminimum nähert sich den Temperaturwerten der Nordsee und erreicht sie auch, falls der im Stavning-Fjord in Dänemark gefundene Standort von T. americana beständig ist, und sie nicht zufällig dorthin getrieben ist.

Die Feststellung von König (1959), dass die Diatomeen des Wattenmeeres im Trockenzu- stand unter dem Einfluss grosser Salzgehaltvariationen stehen, ist ökologisch bedeutsam. Die fortpflanzungsfähigen marinen Diatomeen sind unter diesen Umständen euryhalin. Es soll noch erwähnt werden, dass die ökologischen Beobachtungen der tropischen Gebiete die maximalen Wachstumstemperaturwerte der Terpsinoe americana vertreten. Solche sind u.a. die Wachstumsumgebungen in West-Afrika in der Nähe des Äquators (Woodhead und Tweed 1958 und 1960).

\section{Terpsinoe americana in den Sedimenten der finnischen Teile der Ostsee}

Das Vorkommen von Terpsinoe americana ist bei den biostratigraphischen Beobachtungen verbunden mit dem Litorinameer in der Entwicklungsgeschichte der Ostsee. Auf der Karte, Abb. 5 , ist die Lage von vier Probeserien (6/69, 7/68, 5/72 und 7/72) dargestellt. Ihre Diatomeenstratigraphie wird im folgenden näher untersucht um die Begleitflora von Terpsinoe americana zu erläutern. Auf der Karte sind 33 aus dem Archipel Südwestfinnlands gebohrte Probeserien als Quadrate oder Punkte zu sehen, von denen 462 Proben diatomeenanalytisch untersucht worden sind (vgl. auch Heino 1973 und 1979). Man hat Terpsinoe americana aus 20 verschiedenen Probeserien (die Quadrate) gefunden. Die Gesamtmenge war 23 Einzelwesen oder Fragmente.

In einem aus dem Meergebiet vor der Stadt Naantali gebohrten Profil 6/69 (Diatomeendiagramm, Abb. 6) kommt Terpsinoe americana nur als Einzelfund im oberen Teil der Probeserie im Litorinameerton in $30 \mathrm{~cm}$ Sedimenttiefe vor. Dominante Begleitdiatomeen sind u.a. die marinen Melosira moniliformis, Nitzschia punctata, Cocconeis scutellum, Grammatophora marina und Hyalodiscus scoticus. Diese Arten sind übliche subfossile Diatomeen in den Sedimenten des Litorinameeres (z.B. Mölder und Tynni 1967, 1969 und 1972; Tynni 1980). 


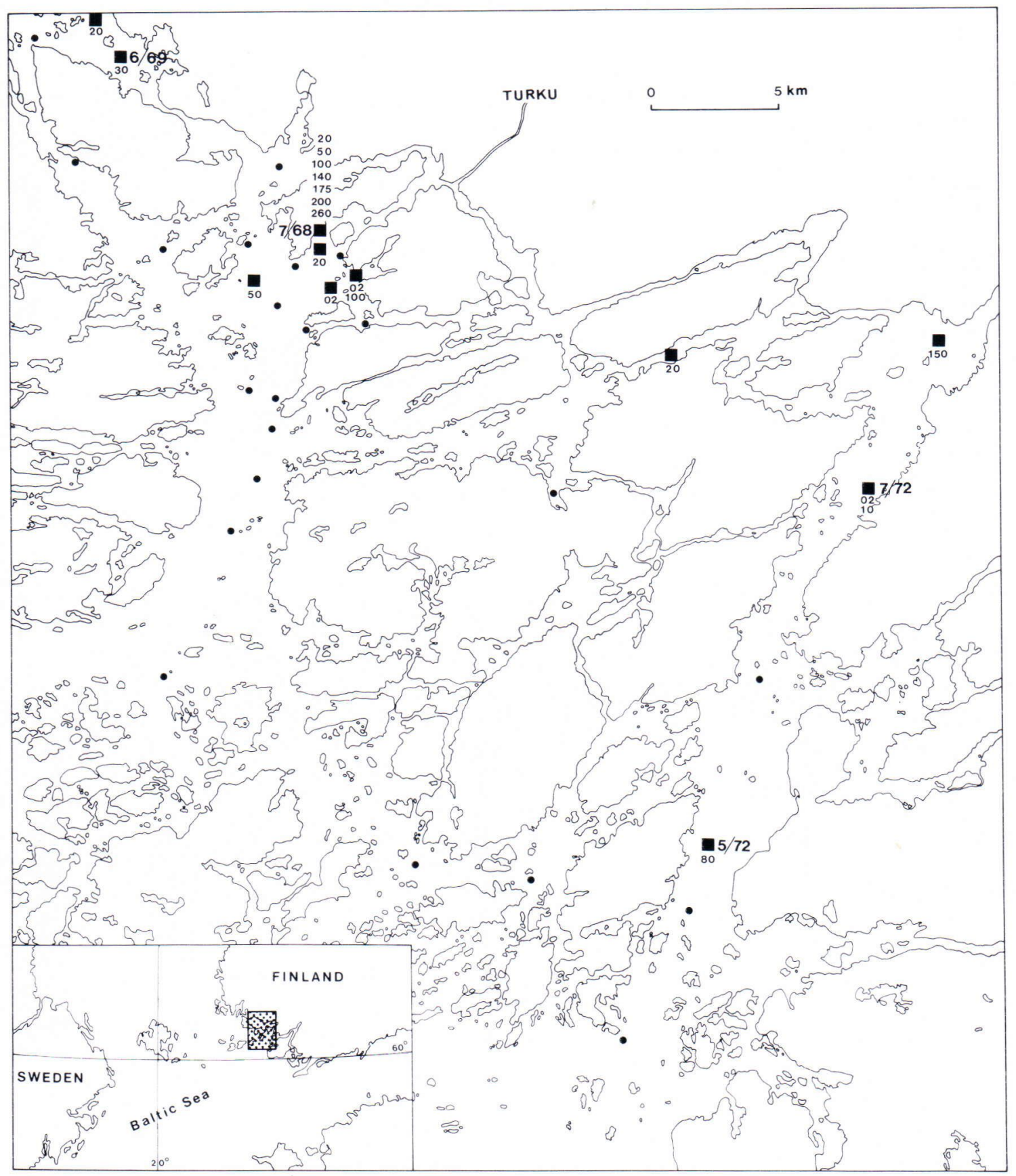

Abb. 5. Die Fundstellen der subfossilen Terpsinoe americana in der südwestfinnischen Schäre (die Quadrate). Die Nummern zeigen die Fundtiefe $(\mathrm{cm})$ in der Lithostratigraphie der Bohrprofile.

Fig. 5. Map showing the distribution of subfossil Terpsinoe americana in the cores from the archipelago of southwestern Finland (the squares). The numbers show the depths $(\mathrm{cm})$ in the lithostratigraphy. 


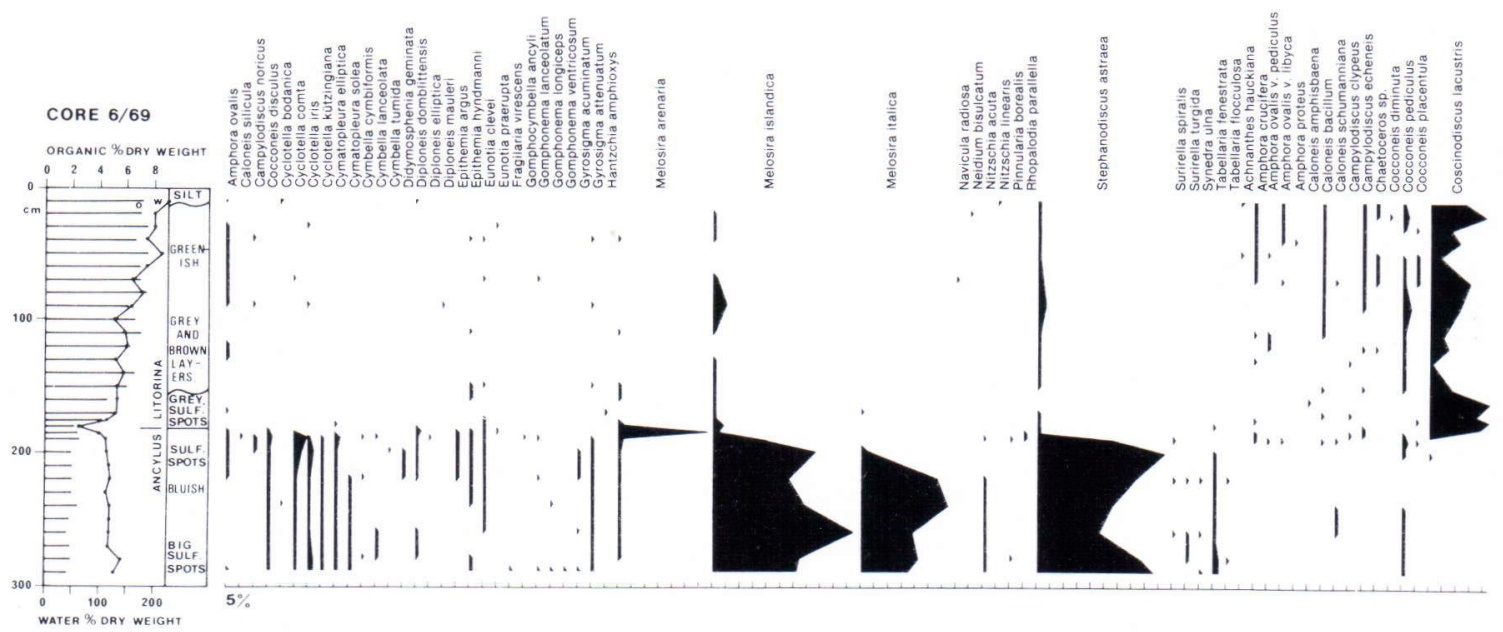

Abb. 6. Die Litho- und Biostratigraphie des Bohrprofils 6/69 (siehe Abb. 5). Die Ancylus/Litorina-Grenze ist diatomeenstratigraphisch deutlich zu sehen. Die Begleitflora von Terpsinoe americana wird näher im Text diskutiert.

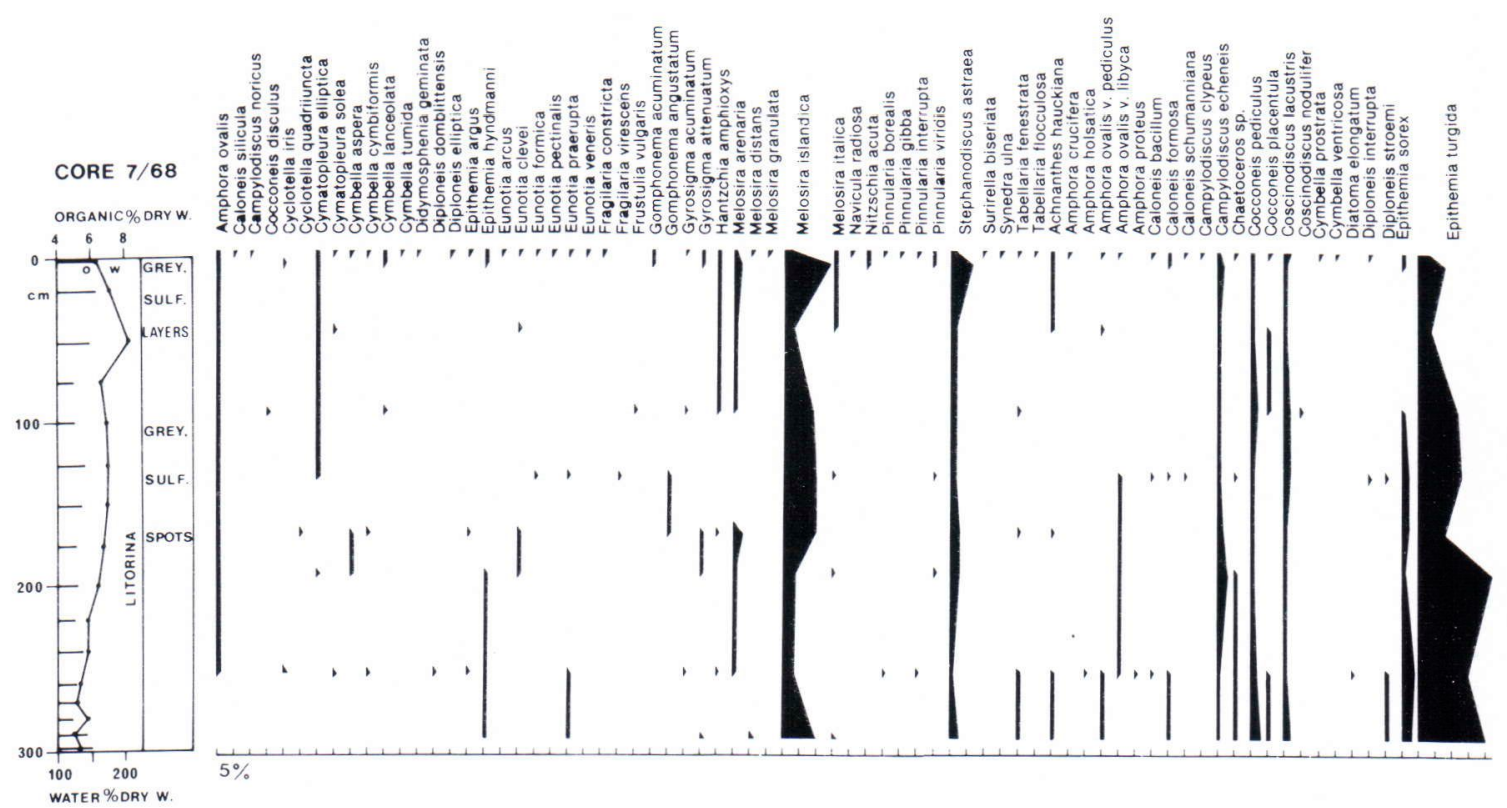

Abb. 7. Die Litho- und Biostratigraphie des Bohrprofils 7/68 (siehe Abb. 5). Die Begleitdiatomeen der Kieselalge Terpsinoe americana in den Litorinasedimenten werden näher im Text diskutiert.

In der von Nord-Airisto vor Turku gebohrten Probeserie 7/68 (Abb. 7) ist das Vorkommen von Terpsinoe americana aussergewöhnlich einheitlich. Die übrige Diatomeenflora ist ebenfalls deutlich marin: Melosira moniliformis und Synedra tabulata weiter unten in der Diatomeenstratigraphie und weiter oben ausser diese Arten auch u.a. Rhabdonema arcuatum, R. minutum, 


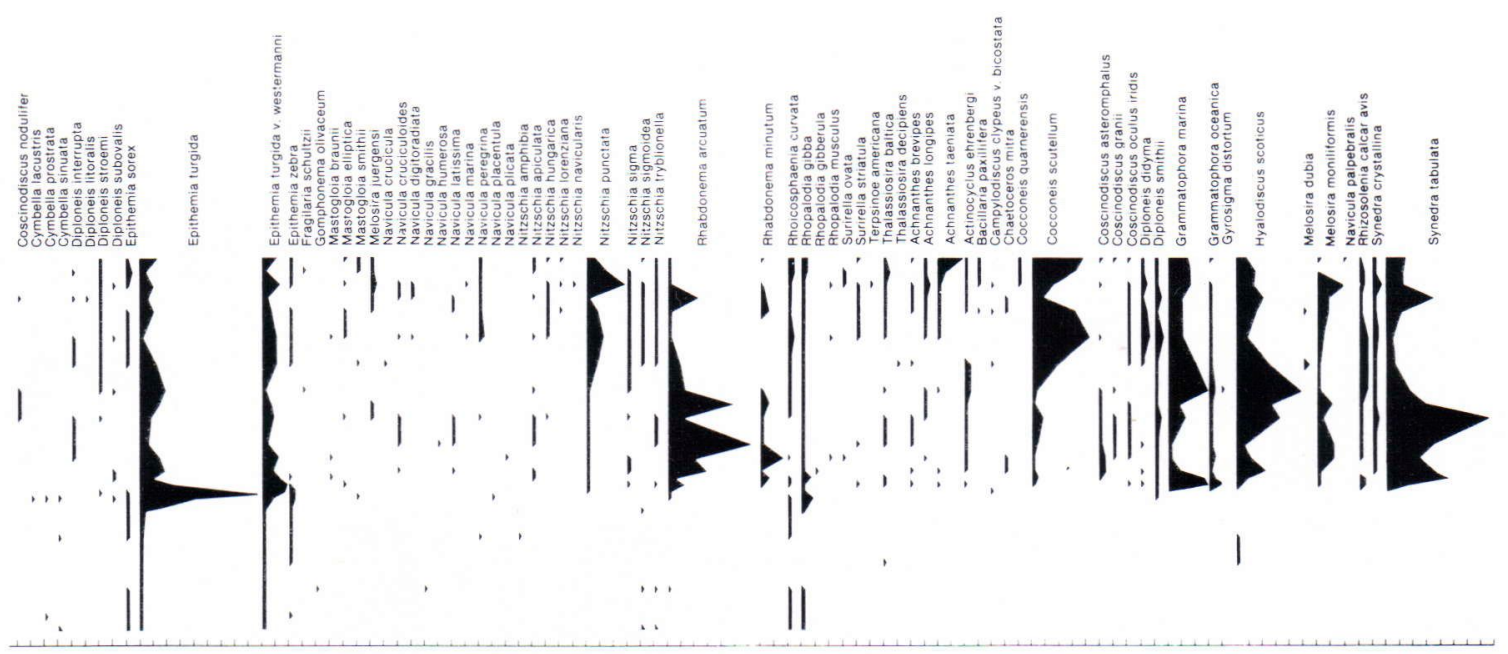

Fig. 6. Litho- and biostratigraphy of the core 6/69 (see Fig. 5). The Ancylus/Litorina limit can be clearly seen in the diatom stratigraphy. For details see text.

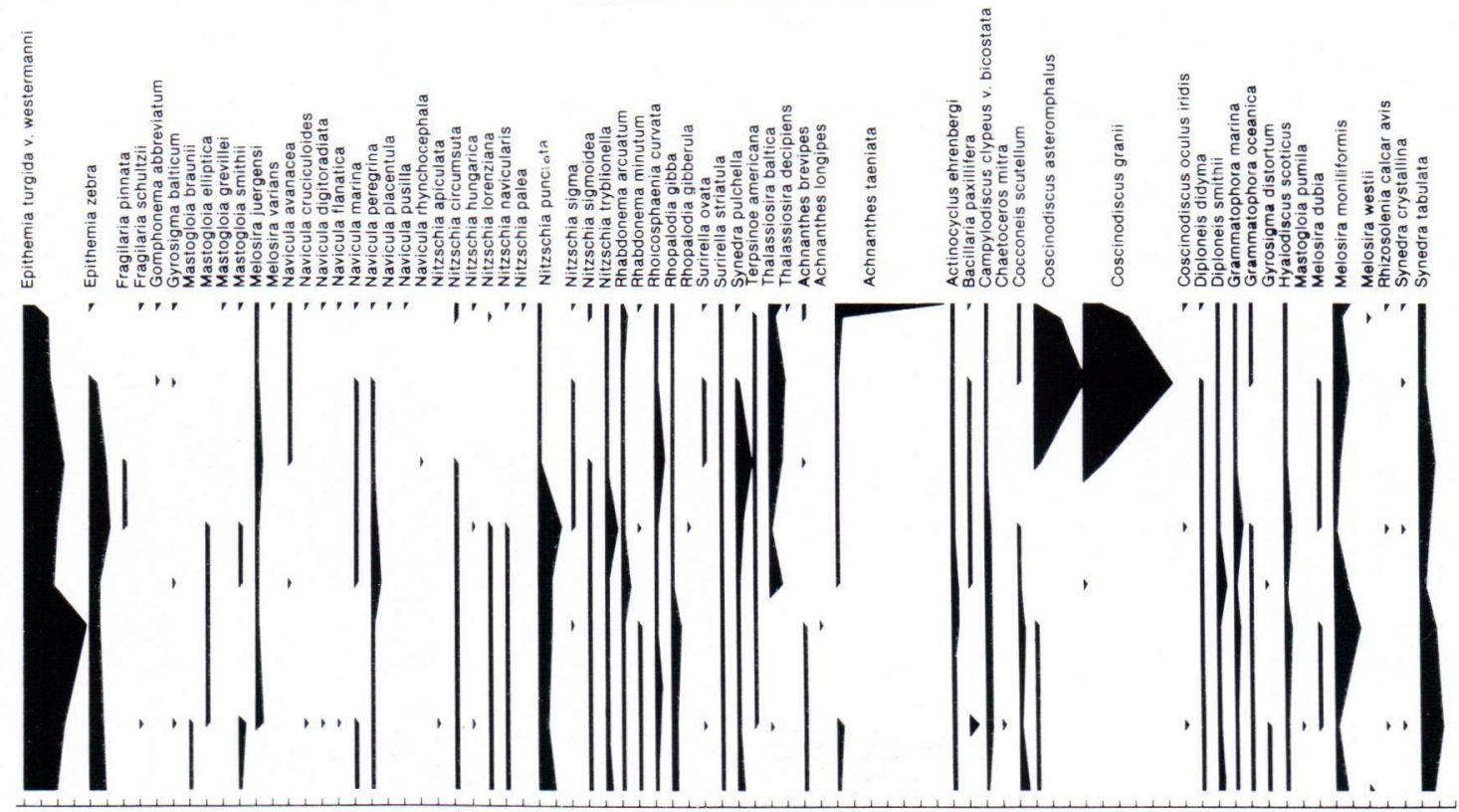

Fig. 7. Litho- and biostratigraphy of the core 7/68 (see Fig. 5). The diatom community with Terpsinoe americana in the Litorinasediments are discussed in details in text.

Synedra pulchella, Thalassiosira baltica und Rhoicosphenia curvata. Höher in der Stratigraphie folgen die planktischen Formen Coscinodiscus asteromphalus und C. granii und ganz an der Oberfläche des Profils kommt Achnanthes taeniata vor. In der Diatomeenstratigraphie sind, obwohl in geringer Zahl, die Grossseeformen Melosira islandica ssp. helvetica und Step- 


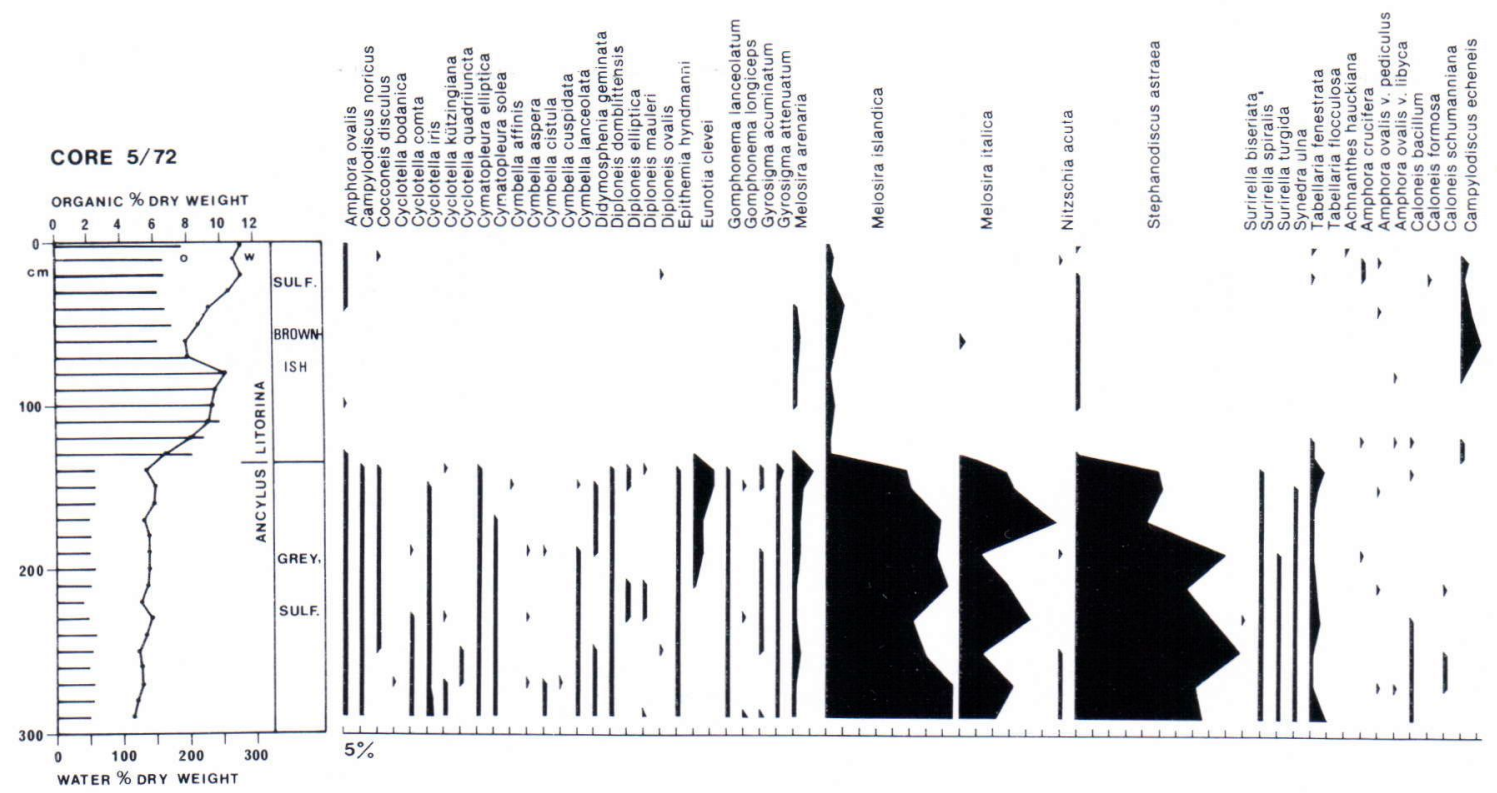

Abb. 8. Die Litho- und Biostratigraphie des Bohrprofils 5/72 (siehe Abb. 5). Terpsinoe americana wurde aus $80 \mathrm{~cm}$ Tiefe im Litorinaton gefunden. Siehe näher im Text.

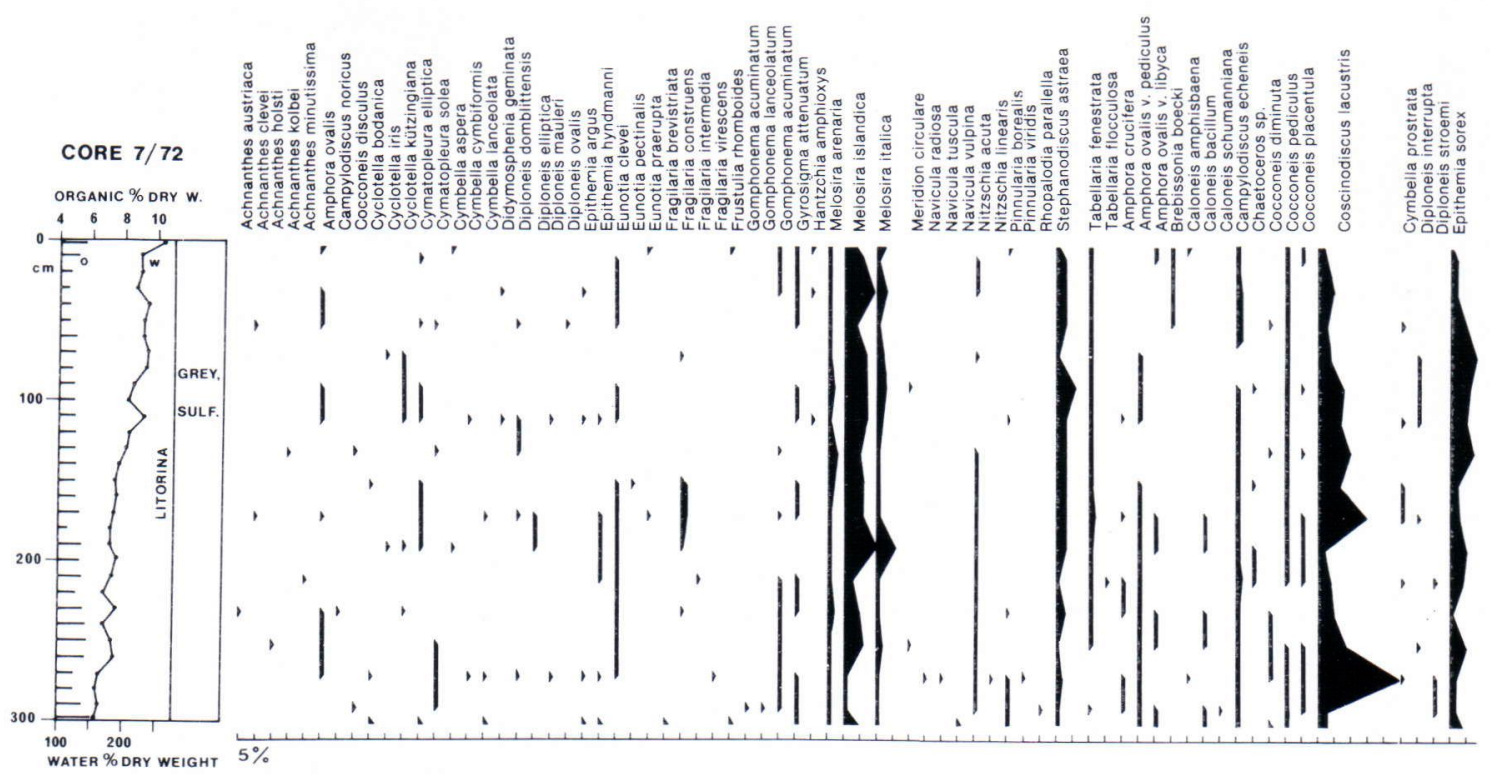

Abb. 9. Die Litho- und Biostratigraphie des Bohrprofils 7/72 (siehe Abb. 5). Die subfossile Diatomeenflora samt Terpsinoe americana ist typisch für das Litorinameer. 


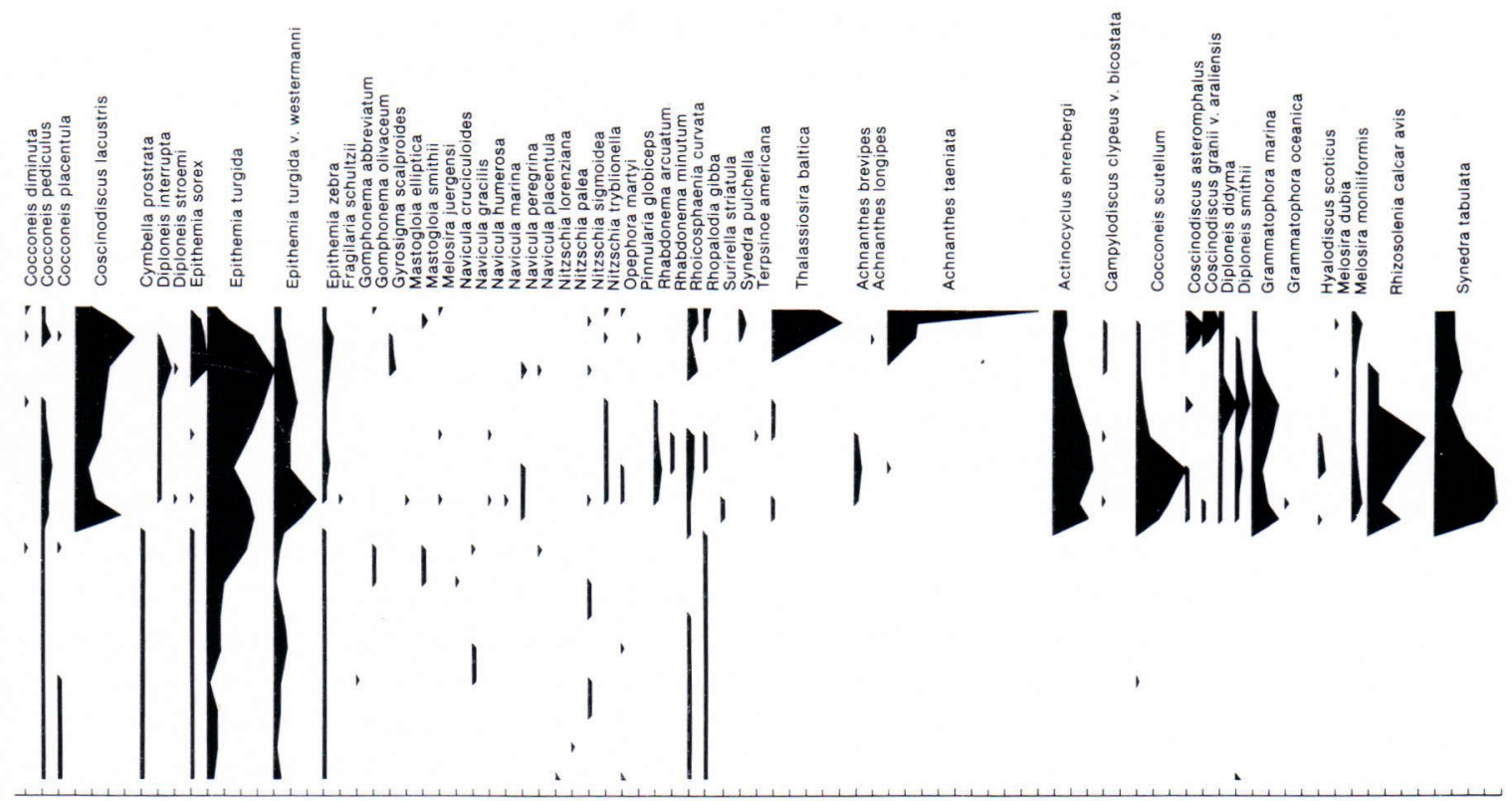

Fig. 8. Litho- and biostratigraphy of the core 5/72 (see Fig. 5). Terpsinoe americana was found from the depth of $80 \mathrm{~cm}$ in the Litorina-clay. For further details see text.

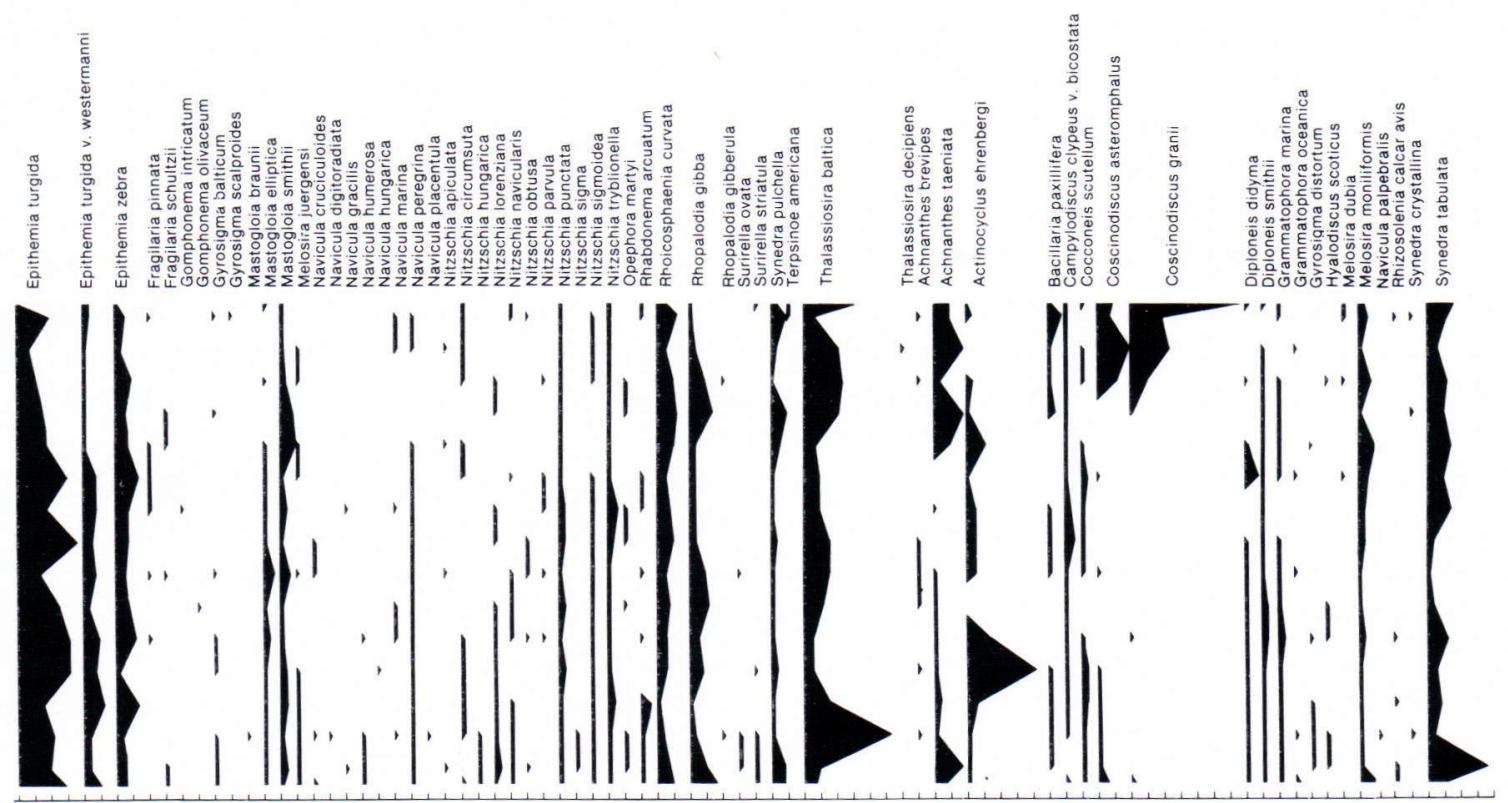

Fig. 9. Litho- and biostratigraphy of the core 7/72 (see Fig. 5). The subfossil diatoms with Terpsinoe americana are typical for the Litorina Sea. 
hanodiscus astraea vertreten; es wird vermutet, dass sie den süssen Einfluss des Flusswassers an der Sedimentierungsstelle abspiegeln. Eine solche mikrofloristische Mischumgebung ist typisch für die finnishe Küstenzone.

In einem in der Paimio Bucht gebohrten Profil 5/72 (Abb. 8) wurde Terpsinoe americana aus $80 \mathrm{~cm}$ Tiefe im Litorinaton gefunden. Die dort vorkommenden anderen Arten sind typisch für die Litorinasedimente der finnischen Küstengebiete: Actinocyclus ehrenbergii, Coscinodiscus lacustris, Cocconeis scutellum, Grammatophora marina, Diploneis didyma, D. smithii, Synedra tabulata und Rhizosolenia calcar avis (Mölder und Tynni 1968, 1970 und 1973).

In einem ebenfalls in der Paimio Bucht gebohrten Profil 7/72 (Abb. 9) kommt Terpsinoe americana in den zwei obersten Proben $(2 \mathrm{~cm}$ und $10 \mathrm{~cm}$ ) vor. Die gesamte Biostratigraphie ist typisch für die Diatomeenflora des Litorinameeres. Von anderen in den selben Tiefen vorkommenden Diatomeen können die folgenden erwähnt werden: Thalassiosira baltica, Coscinodiscus granii, Achnanthes taeniata, Synedra tabulata, Bacillaria paxillifera, Melosira moniliformis, und Epithemia turgida. Melosira islandica ssp. helvetica und Stephanodiscus astraea spiegeln vermutlich auch in dieser Probeserie einem im Flusswasserrezipient vorkommenden geringen Süsswassereinfluss wieder.

Ausser den voran beschriebenen Fundstellen wurden zwei andere Lithostratigraphien aus Finnland dargestellt, wo Terpsinoe americana als Einzelfund in marinen Diatomeenassoziationen vorkommt. Alhonen (1974) hat im Zusammenhang mit einem vorgeschichtlichen Netzfund in Tuorsniemi in der Nähe von Pori die Diatomeenstratigraphie der Fundstelle untersucht und festgestellt, dass Terpsinoe americana in der oberen Siltschicht vorkommt (Alhonen 1974, Abb. 3). Campylodiscus echeneis, Thalassiosira baltica, Nitzschia punctata und Epithemia turgida waren dominierende Arten in derselben Assoziation. Die Netzschwimmer kamen jedoch im tieferen Tonsediment vor, wo Rhab- donema arcuatum die überwiegend dominanteste Diatomee (Maximum 95 \%) war. Das Alter der Netzschwimmer ist $3850 \pm 110$ B.P., sodass Terpsinoe americana in diesem Profil deutlich jünger als die Schwimmer ist. Es soll noch erwähnt werden, dass an dieser Stelle auch Knochen einer Sattelrobbe (Phoca groenlandi$c a$ ) gefunden wurden, eine Tatsache, die auf das Litorinameer hindeutet (Forsten und Alhonen 1975).

Eronen, Heikkinen und Tikkanen (1982) haben eine Diatomeenstratigraphie, die die Entwicklung des Sees Pyhäjärvi in Satakunta in Südwestfinnland nach der letzten Eiszeit erläutert, dargestellt. Dort kommt Terpsinoe americana in einer marinen Tongyttjaablagerung vor der Abschnürung des Sees Pyhäjärvi vor. Ausserdem wird die Diatomeenassoziation durch u.a. Melosira moniliformis, Grammatophora oceanica, Synedra tabulata, Cocconeis scutellum, Diploneis smithii, Coscinodiscus lacustris und Epithemia turgida charakterisiert (Eronen et al. 1982, Abb. 8 und 9). Die Wachstumsumgebung der Diatomeenflora deutet auf eine Bucht des Litorinameeres hin.

\section{Diskussion}

Die rezenten Beobachtungen über die Ökologie der Terpsinoe americana deuten auf eine marine Litoralumgebung wie schon festgestellt wurde. Die Art ist polyhalob, aber kann auch im Brackwasser wachsen. Sie ist eine übliche Diatomee in den Küstenzonen tropischer Gewässer, aber wächst ebenfalls in gemässigten Meeresumgebungen, und in beiden als Epiphytform. In der Entwicklungsgeschichte der Ostsee ist Terpsinoe americana jedoch selten. Sporadisch erscheint sie zwar in manchen Probeserien gleichmässig während der ganzen Litorinameerperiode, aber kaum danach. Wir haben die in der Geschichte der Ostee nur als Subfossil vorkommende Terpsinoe americana als eine primäre im Litorinameer wachsende Diatomee behandelt. Ihre Fundstellen liegen jedoch an den 
Flussmündungen, eine Tatsache, die die rezenten Beobachtungen unterstützen. Die Art ist ja nur auf Estuarien gefunden worden. Anders interpretiert könnte die lokale Lage an der finnischen Küstenzone darauf hindeuten, dass die Schalen von Terpsinoe americana aus älteren Sedimenten stammten und sich aufs neue sekundär an den jetztigen Fundstellen sedimentiert hätten. Alhonen und Mansikkaniemi (1978) haben nämlich festgestellt, dass aufgrund der Flusserosion im Flussseston, je nach dem Probenahmezeitpunkt, sogar $15 \%$ aufs neue sedimentierte marine und Brackwasserdiatomeen vorkommen können. In unserem Material kommt Terpsinoe americana sowohl in der Benthos-Epiphytenassoziation als zusammen mit den planktischen Formen vor, wobei das Nachweisen der primären Wachstumsstelle mit Hilfe der Begleitflora nicht eindeutig ist. Aufgrund der Lithostratigraphie von Tuorsniemi (Alhonen 1974) ist die Art dort wahrscheinlich als Epiphyt auf dem Siltgrund gewachsen, was auch durch rezente ökologische Beobachtungen bestätigt wird (z.B. Riznyk 1973).

Wie weit das stratigraphische Vorkommen von Terpsinoe americana die thermische Hydrodynamik und die Paläosalinität des Küstenfazies des Litorinameeres erläutert, ist natürlich eine äusserst interessante aber mehrdeutige Frage. Sie ist jedenfalls ein deutlicher Indikator der gesamten ökologischen Parameter. Als typische ozeanische Art kann Terpsinoe americana als Vertreter des Höhepunkts der Salinität und der thermischen Entwicklung des Litorinameeres betrachtet werden, sie verschwindet am Ende dieser Entwicklungsperiode der Ostsee. Es soll in Betracht genommen werden, dass Terpsinoe americana in der Geschichte der Ostsee an ihrem ökologischen Grenzgebiet lebt, was ihren verhältnismässig geringen Anteil in der Diatomeenflora des Litorinameeres erklären könnte. Ihr Verschwinden aus der Ostsee hängt jedenfalls mit der Abnahme der Salinität und der Abkühlung des Klimas nach dem Ende der postglazialen Wärmezeit zusammen.
Zum Schluss ist es interessant die stratigraphische Geschichte von Terpsinoe americana an ihren anderen Wachstumsumgebungen zu erläutern. In Nord-Amerika gehört die Gattung Terpsinoe zu den aus dem Moreno-Tonschiefer gefundenen ältesten kreidezeitlichen Diatomeen des Kontinents, wie auch die Gattungen Anaulus, Biddulphia. Eunotogramma und Triceratium (Long et al. 1946 und Wornardt 1972). Es soll noch erwähnt werden, dass Terpsinoe americana zu den extinktischen spätkreidezeitlichen Arten gehört. Terpsinoe americana ist schon recht früh in der Diatomeenflora aufgetreten, und ihre Fossilbeobachtungen sind häufiger als diejenigen der anderen Terpsinoe-Arten. Andrews (1976) stellt fest, dass die frühsten Terpsinoe americana Funde in Nord-Amerika erst aus dem Miozän stammen. Die Datierung basiert u.a. auf die Choptank-Formation in Maryland. Terpsinoe americana kam sehr häufig in der Kieselguhr aus Churchill County in Nevada vor. Diese Ablagerung wurde entweder im oberen Miozän oder im unteren Pliozän sedimentiert (Okuno 1959). In einem japanischen Material (Ichikawa et al. 1955) stammt die Art, zwar var. macrocephala, aus dem Miozän. Tertiäre Beobachtungen gibt es ausserdem aus dem See Obuchi (Hasegawa 1976) und aus Oamary in Neu Zealand (Crosby und Wood 1959).

In Europa kommt Terpsinoe americana fossil in den Ablagerungen von Burdigalia in Spanien vor, sie sind am Ende das Oligozäns sedimentiert; wie auch in den Moronitas-Sedimenten bei Sevilla (Colom 1952). Die letztgenannten sind in einem wärmeren Meer als das heutige Mittelmeer abgelagert. Ein gewisser Teil der benthonischen Moronitas-Flora ist dieselbe wie in der Gezeitenzone der europäischen Atlantik Küste (Colom 1952). Systematisch diskutiert sind Terpsinoe americana f. trigona Pant. bei Zagreb (Jurilj 1957) aus dem oberen Miozän und $T$. americana var. trigona Grun. in der CeyssacAblagerung aus dem Pliozän (Lauby 1919). Die erstgenannte ist nach Cleve-Euler (1915) Triceratium americana. Nach Schmidts Atlas (Taf. 
76, Fig. 28 und Taf. 159, Fig. 1, 2) unterscheidet sich Terpsinoe americana $f$. trigona deutlich von Triceratium americana.

In der Sowjetunion ist die Gattung Terpsinoe nicht sehr üblich in den marinen tertiären Ablagerungen, man kennt sie jedoch aus dem Miozän (Gleser et al. 1974, Taf. 41, Fig. 1, 2). Terpsinoe americana gehört auch zur Diatomeenflora des Litorinameeres in den Ostseegebieten der Sowjetunion. Während dem Wärmeoptimum

\section{Literaturverzeichnis}

Aario, L., 1932. Pflanzentopographische und paläogeographische Mooruntersuchungen in N-Satakunta. Fennia 55, 1; Comm. Inst. Forest. Fennicae 17, 1.

Alhonen, P., 1974. Stratigraphy and palaeoecology of the net-find site at Tuorsniemi, Pori, Western Finland. Karhunhammas 1, 8-13.

Alhonen, P. \& Mansikkaniemi, H., 1978. Observations of sedimentation of diatoms in some Finnish rivers. Fennia 156, $1-9$.

Amosse, M. A., 1924. Diatomées de la Orientale d'Afrique. Bull. Mus. Nat. d'Histoire Naturelle Nr. 1, 109-118, Nr. 2, 159-166, Nr. 3, 247-254, Nr. 4, 329-335.

Andrews, G. W., 1976. Miocene marine diatoms from the Choptank Formation, Calvert County, Maryland. Geol. Surv. Profess. Paper 910, 26 p, 7 pls.

Boyer, C. S., 1916. The Diatomaceae of Philadelphia and vicinity. Philadelphia

-, 1926/7. Synopsis of North American Diatomaceae. Proc. Acad. Nat. Sci. 78 \& 79.

Brander, G., 1937. Ein Interglazialfund bei Rouhiala in Südfinnland. Bull. Comm. Géol. Finlande 118, 76 p.

Brockmann, C., 1928. Die Diatomeen in marinen Quartär Hollands. Abhandl. Senckenberg. Naturfors. Gesell. 41, 117-187, 4 Taf.

—, 1940. Diatomeen als Leitfossilien in Küstenablagerungen. Westküste 2, 150-181.

-, 1950. Die Watt-Diatomeen der schleswig-holsteinischen Westküste. Abhandl. Senckenberg. Naturfors. Gesell. $478,1-26$.

Brun, J., 1891. Diatómees Marines, Fossiles ou Pélagiques. Memoires Soc. Phys. et d'Hist. Naturelle Geneve, t. 31 (1), $1-47$.

Cholnoky, B. J., 1968. Die Ökologie der Diatomeen in Binnengewässern. 8, 700 p. J. Cramer, 3301 Lehre.

Cleve, P. T., 1881. On some new and little known Diatoms. Kongl. Svenska Vetenskaps-Akademiens Handl. Bd. 18 (5), $1-28$. des Holozäns kam T. americana recht häufig an der Nordseeküste vor, hat sich aber bei der Abkühlung des Klimas deutlich verringert. Dieser Zug erinnert an die Geschichte ihres stratigraphischen Vorkommens in der Ostsee.

Danken. Herrn Forschungsassistent Kalevi Hokkanen danken die Verfasser bestens für seine vielseitige Hilfe und Frau Dr. Säde Mantere-Alhonen für die Übersetzung des Manuskripts ins Deutsche.

Cleve-Euler, A., 1915. New Contributions to the Diatomaceous Flora of Finland. Arkiv f. Botanik, 14 (9), $1-81$.

-, 1951. Die Diatomeen von Schweden und Finnland. Kungl. Svenska Vetenskapsakademiens Handl. Fjärde Serien, Bd. 2, Nr. 1, S. 1-163, Fig. 1-294, 6 Taf.

Colom, G., 1952. Aquitanian-Burdigalian diatom deposits of the North Betit strait, Spain. Journal of Paleontology 26 (6), 867-885.

Crosby, L. H. \& Wood, E. J. F., 1959. Studies on Australia and New Zealand diatoms 2. Normally epontic and benthnic genera. Trans. Royal Soc. New Zealand, Vol. 86 , p. $1-58$.

Eronen, M., Heikkinen, O.\& Tikkanen, M., 1982. Holocene development and present hydrology of Lake Pyhäjärvi in Satakunta, southwestern Finland. Fennia 160, $195-223$.

Fontell, C. W., 1926. Om brack- och saltvattendiatomacéers förekomst i sött vatten i närheten av kusten. Acta Soc. Fauna et Flora Fennica 55, no. 7.

Forsten, A. \& Alhonen, P., 1975. The subfossil seals of Finland and their relation to the history of the Baltic Sea. Boreas 4, 143-155.

Giffen, M. H., 1970. Contributions to the diatom flora of South Africa 4. The Marine Littoral Diatoms of the Estuary of the Kowie River, Port Alfred, Cape Province. Nova Hedwigia 31, 259-312.

Gleser, S. I.; Jousé, A. P.; Makarova, I. V.; ProschkinaLavrenko, A. I. (Editor-in-Chief) \& Sheshukova-Poretzkaja, V. S., 1974: The diatoms of the USSR, fossil and recent. Publishing House »Nauka», Leningrad branch $404 \mathrm{~S}$.

Hagelstein, R., 1938. The Diatomaceae of Porto Rico and the Virgin Islands. New York Academy of Sciences, Scientific Surv. Porto Rico and the Virgin Islands, Vol. 8, P. 3, 313-450.

Hasegawa, Y., 1976. Fossil diatom composition of Lake Obuchi, Aomori prefecture. Paleolimnology of Lake Biwa and the Japanese Pleistocene, Vol. 4, Ed. S. Horie, 430-498. 
Heino, A., 1973. Bottom deposits and sedimentation in northern Airisto in Southwestern Finland. Bull. Geol. Soc. Finland 45, 131-142.

,- 1979 . The pattern of organic content and some heavy metals in the bottom sediments of Airisto, SW-Finland. Turun yliopiston maantieteen laitoksen julkaisuja - Publicationes Instituti Geographici Universitatis Turkuensis $89,1-52$.

Hustedt, F., 1927-30. Die Kieselalgen Deutschlands, Österreichs und der Schweiz. i Teil. Rabenhorst: Kryptogamenflora, 7. Leipzig.

-, 1955. Marine littoral diatoms of Beaufort, North Carolina. Duke University Marine Station, Bull. 6, 68 S.

Hyyppä, E., 1937. Postglacial changes of shore-line in South Finland. Bull. Comm. gèol. Finlande 120, 225 S.

Ichikawa, W.; Kaseno, Y. \& Kojima, K., 1955. On the Miocene nonmarine diatomite in the vicinity of Nakajimamachi. The Journal of the Geolog. Soc. of Japan, Vol. 61, No. 719, 381-386.

Jurilj, A., 1957. Dijatomeje Sarmatskog mora okoline Zagreba/Flora of Diatoms of Sarmatic Sea in Environs of Zagreb. Jugoslavenska Akad. Znanosti i Umjetnosti. Acta biologica I, 5-154.

König, D., 1959. Diatomeen der Bucht von Arcachon (Dép. Gironde, Frankreich). Z.deutsch.geol.Ges. Bd. 111, 1. Teil, 35-61.

Lauby, A., 1910. Recherches Paléophytologiques dans le Massif Central. Bull. Serv. Carte Géol. France 125, (20) p. $1-398,14 \mathrm{pl}$.

Lohman, K. E., 1938. Pliocene diatoms from the Kettleman Hills, California. U.S. Geol. Surv. Prof. Pap. 189-C, 79-102.

Long, J. A., Fuge, D. P. \& Smith, J., 1946. Diatoms of the Moreno Shale. Journ. Paleontology Vol. 20, Nr. 2, 89-118.

Mann, A., 1907. Report on the Diatoms of the Albatross Voyages in the Pacific Ocean, 1888-1904. Contrib. U.S. Nat. Herb., Bd. 10, pt. 5, 221-442.

Moreira, F. H., 1960. Diatomaceas no trato digestivo da Tegula viridula Gmelin. (Chrysophyta-Bacillariophyceae). Bol. Univ. Parana, Bracil. Botanica 1, 27 p. Curitiba.

Mölder, K. \& Tynni, R., 1967. Über Finnlands rezente und subfossile Diatomeen I. C.R. Soc. geol. Finlande 39, 199-217.

—, 1969. Über Finnlands rezente und subfossile Diatomeen III. Bull. Geol. Soc. Finland 41, 235-251.

—, 1970. Über Finnlands rezente und subfossile Diatomeen IV. Bull. Geol. Soc. Finland 42, 129-144.

-, 1972. Über Finnlands rezente und subfossile Diatomeen VI. Bull. Geol. Soc. Finland 44, 141-149.

-, 1973. Über Finnlands rezente und subfossile Diatomeen VII. Bull. Geol. Soc. Finland 45, 159-179.

Okuno, H., 1959. 375. Electron-microscopic fine structure of fossil diatoms. 6. Stereoscopic observations. Trans. Proc. Palaeont. Soc. Japan, N.S. No. 36, pp. 185-191.

Peragallo, H. et M., 1897-1907. Diátomees Marines de France et des Districts Marimes Voisins. Grez-sur Loing, $491 \mathrm{p}$.

Proshkina-Lavrenko, A. I., 1949. Diatomovyi Analiz (edit.). 1, 1-239, Moscow-Leningrad.

Riznyk, R. Z., 1973: Interstitial Diatoms from two tidal flats in Yaquina Estuary, Oregon, U.S.A. Botanica Marina $16,113-138$.

Ross, R. \& Sims, P. A., 1971. Generic limits in the Biddulphiaceae as indicated by the scanning electron microscope. In Scanning Electron'Microscopy: Systematic and Evolutionary Applications (Heywood, V.H., editor). London and New York.

,- 1972 . The fine structure of the frustule in centric diatoms: a suggested terminology. Br. phycol. J. 7, 139 -163 .

Schmidt, A., 1874-1959. Atlas der Diatomaceen-Kunde, founded by A. Schmidt, continued by M. Schmidt, F. Fricke, O. Müller, H. Heiden and F. Hustedt. Aschersleben-Leipzig-Berlin.

Simonsen, R., 1962. Untersuchungen zur Systematik und Ökologie der Bodendiatomeen der westlichen Ostsee. Int. Rev. gesamt. Hydrobiol. System. Beih. 1, 144 p.

-, 1965. Ökologische Bemerkungen zu der tropischen Kieselalge Hydrosera triquetra Wallich und zur Aerophilie der Diatomeen. Int. Rev. Hydrobiol. 50, 1, S. $49-56$.

,- 1972 . Ideas for a more natural system of the centric diatoms. Nova Hedwigia, Beih. 39, 37-54.

,- 1979 . The diatom system: Ideas on Phylogeny. Bacillaria $2,9-71$.

Taylor, F. J., 1970. A preliminary annotated check list of diatoms from New Zealand coastal waters. Trans. Roy. Soc. New Zealand Biol. Sci. Vol. 12, (14), 153-174.

Tynni, R., 1977. Hydrosera trifoliata und ihre Mutationsformen. Comm. Biol. 90, 1-12. Helsinki.

-, 1980. Über Finnlands rezente und subfossile Diatomeen, XI. Geological Survey of Finland, Bulletin 312, $1-93$.

Wood, E. J. F., 1963. A study of the diatom flora of fresh sediments of the South Texas bays and adjacent waters. Pub. Inst. mar. sci. univ. Texas, vol. 9, 237-310.

Woodhead, N. \& Tweed, R. D., 1958. A check list of tropical West African algae. Hydrobiologia 11 (3-4), 299 -395 .

-, 1960. A second check-list of tropical West African algae. Hydrobiologia 15 (3), 225-286.

Wornardt, Jr. W. W., 1972. Stratigraphic distribution of diatom genera in marine sediments in Western North America. Palaeography, Palaeoclimatology, Palaeoecology. Elsevier Publ. Comp., Amsterdam, 49-74.

Manuscript received, January 4, 1984. 